\title{
XXVI. On the ratio between the mean spherical and the mean horizontal candle-power of incandescent electric lamps
}

\section{J.A. Fleming M.A. D.Sc. F.R.S.}

To cite this article: J.A. Fleming M.A. D.Sc. F.R.S. (1905) XXVI. On the ratio between the mean spherical and the mean horizontal candle-power of incandescent electric lamps, Philosophical Magazine Series 6, 10:56, 208-216, DOI: 10.1080/14786440509463364

To link to this article: http://dx.doi.org/10.1080/14786440509463364

册Published online: 16 Apr 2009.

Submit your article to this journal $[\pi$

Џll Article views: 2

Q View related articles $₫$ 
to $\cdot 64 v_{0}$. From this it can at once be deduced that 48 of the total energy of the a particle, shot out by radium itself, is absorbed when it ceases to ionize the gas. Now it can be calculated from the heating effect of radium at its minimum activity-25 gram-calories per hour per gramthat the kinetic energy of the $\alpha$ particle is $4.7 \times 10^{-6} \mathrm{erg}$. The amount of energy absorbed when the $\alpha$ particles just cease to ionize the gas is $2 \cdot 3 \times 10^{-6} \mathrm{erg}$. Assuming that this energy is used up in ionization, and remembering that the $\alpha$ particle from radium itself produces 86,000 ions, the average energy required to produce an ion is $2.7 \times 10^{-11} \mathrm{erg}$. This is equivalent to the energy acquired by an ion moving freely between two points differing in potential by 24 volts.

McGill University, Montreal, May $1,1905$.

XXVI. On the Ratio between the Mean Spherical and the Mean Horizontal Candle-Power of Incandescent Electric Lamps. By J. A. Fleming, M.A., D.Sc., F.R.S., Professor of Electrical Engineering in University College, London*.

A PAPER was real before the Physical Society on A November 11th, $1904 t$, by Mr. G. B. Dyke, B.Sc., in which, amongst other matters, he gave the results of numerous measurements made in the Pender Electrical Laboratory of University College, London, of the ratio of the mean spherical candle-power of incandescent electric lamps to the mean horizontal candle-power, taken when the lamp was rotating round a vertical axis. In all cases the ratio of the mean spherical to mean horizontal candle-power was experimentally found to be a number near to 0.78 for about nine different types of electric glow-lamps. This constant ratio must depend upon definite optical facts, and cannot be a matter of accident. That this is the case can be shown by considering a typical instance.

Let us suppose a short straight filament $a b$ (fig. 1) rendered incandescent to be placed vertically in the centre of a sphere described by the revolution of a semicircle $\mathrm{PHQ}$ round a diameter $\mathrm{P} Q$, coinciding with the direction of the filament. Let the length of the filament be small compared with the radius $F B=r$ of the sphere. Let $I_{H}$ represent the

* Communicated by the I'hysical Society : read June 16, 1905.

+ See Mr. G. B. Dylke, "On the Practical Determination of the Mean Spherical Candle-Power of Incandescent and Arc Lamps," Phil. Mag. ser. (f, vol, ix. p. 136, Jan. 1905 ; also Proc. Phys. Soc. vol. xix. p. 399 (19)5). 
Horizontal Candie-Puwer of Ineandescent Lamps. 209 intensity of the light emitted or the candle-power of the filament in a horizontal direction $\mathrm{FH}$, and let $\mathrm{I}$ be the intensity in any direction $\mathrm{NN}$ making an angle $\mathrm{NFH}=\theta$ with the horizontal direction. Let $\mathrm{I}_{\mathrm{S}}$ be the mean spherical candle-power.

\section{Fig. 1.}

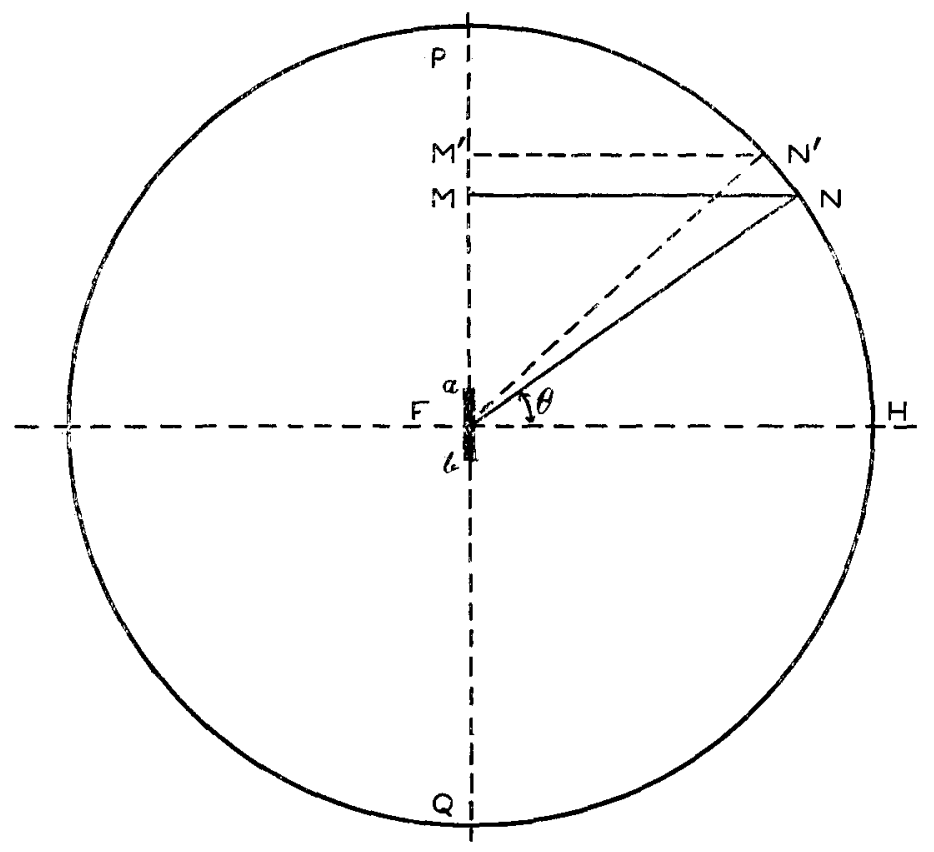

Let us then start with the assumption that $I=I_{H} \cos \theta$. The justitication for this is found in the fact that if a long straight incandescent filament is placed behind a small rectangularshaped hole in a metallic plate, then inclining the filament to the plane of the plate within certain angular limits makes no sensible difference in the quantity of light which comes through the hole*. Consider then a zone of the sphere swept out by an elementary arc $\mathrm{NN}^{\prime}$ of the semicircle. The surface of this zone is $2 \pi r^{2} \cos \theta d \theta$, where $d \theta$ denotes the angle $\mathrm{N}^{\prime} \mathrm{FN}$.

Hence the quantity of light falling on the whole zone is $\mathrm{I}_{\mathrm{H}} 2 \pi r^{2} \cos ^{2} \theta d \theta$, and the total quantity of light incident on the

* For the limits within which the above statement is confirmed by experiment the reader is referred to the Appendix to this Paper.

Phil. Mug. S. 6. Vol. 10. No. 56. Aug. 1905. P 
entire surface of the circumscribing sphere is

$$
4 \pi r^{2} \mathrm{I}_{\mathbf{H}} \int_{0}^{\frac{\pi}{2}} \cos ^{2} \theta d \theta
$$

Hence by the definition of mean spherical candle-power $\mathrm{I}_{\mathrm{s}}$ we have

$$
4 \pi r^{2} \mathrm{I}_{\mathrm{S}}=4 \pi r^{2} \mathrm{I}_{\mathrm{H}} \int_{0}^{\frac{\pi}{2}} \cos ^{2} \theta d \theta
$$

or

$$
\frac{\mathrm{I}_{\mathrm{S}}}{\mathrm{I}_{\mathrm{II}}}=\int_{0}^{\pi} \cos ^{2} \theta d \theta=\int_{0}^{\frac{\pi}{2}}\left(\frac{1+\cos 2 \theta}{2}\right) d \theta=\frac{\pi}{4}=0.785 .
$$

Accordingly for such a short straight filament the ratio experimentally found by $\mathrm{Mr}$. Dyke, viz., $\mathrm{I}_{\mathrm{S}} / \mathrm{I}_{\mathrm{II}}=0 \cdot 78$, holds good.

Conversely, the fact that experiment shows that for any incandescent lamp $I_{S} / I_{H}=\pi / 4$ nearly, is a proof that the cosine law of luminous radiation from the filaments in different direction holds good, with considerable exactness.

The departure from the above rule when the filament is not short compared with the radius of the sphere can be found as follows:-

Consider a straight incandescent filament of length $2 l$ the half of which is represented by the line $A B$ (see fig. 2) placed

Fig. 2.

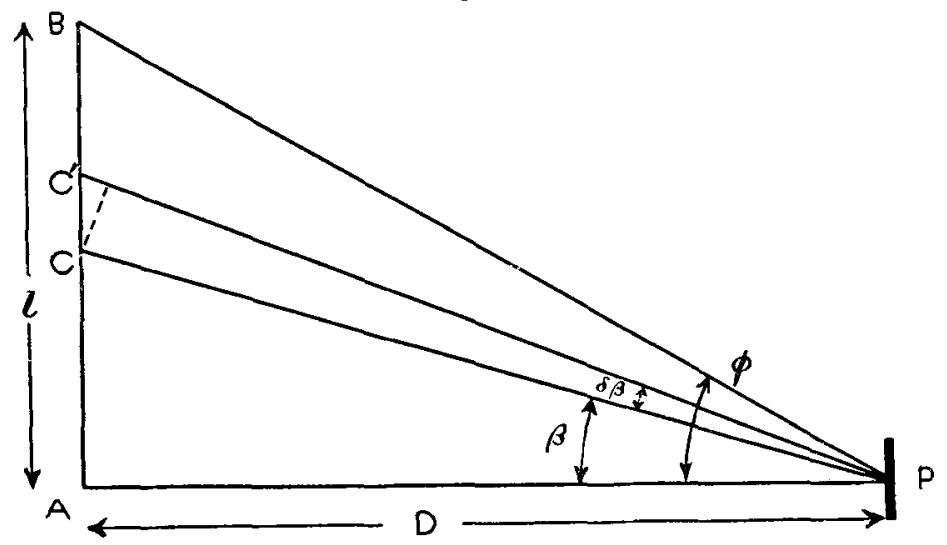

at a distance $\mathrm{AP}=\mathrm{D}$ from a photometer-disk $\mathrm{P}$, the filament being parallel to the photometer-plane. Let an element of length at $C$ be denoted by $d l$. Let the angle APB subtended at 
$P$ by half the length of the filament be denoted by $\phi$, and let the angle APC be denoted by $\beta$. Let the intensity of the light sent out or the candle-power per unit of length of the filament in a direction normal to itself be denoted by $I_{H}$, then the illumination on the photometer-disk, assuming it to follow the above mentioned cosine law, due to an element $d l$ of the filament at $\mathrm{C}$ subtending an angle $d \beta$ at $\mathrm{P}$ will be given by the expression

$$
\frac{I_{\mathrm{H}} \cos ^{2} \beta d l}{(\mathrm{C} P)^{2}}=\frac{\mathrm{I}_{\mathrm{H}} \cos ^{4} \beta d l}{D^{2}} .
$$

Also from the geometry of the figure we have

$$
d l=\frac{\mathrm{D} \cdot d \beta}{\cos ^{2} \beta} .
$$

Hence the illumination on the photometer-disk due to the element $d l$ of the filament at $C$ is given by the expression

$$
\frac{\mathrm{I}_{\mathrm{H}} \mathrm{D} \cdot \cos ^{2} \beta d \beta}{\mathrm{D}^{2}} \text {. }
$$

This is the same illumination as that which would be given by a vertical element of length $D d \beta$ placed at $A$, but having a horizontal intensity or horizontal candle-power per unit of length equal to $I_{H} \cos ^{2} \beta$.

To obtain the whole illumination on the photometer-disk due to the whole filament of length $2 l$, we have to integrate the above expression for one element, between the limits $\beta=0$ and $\beta=\phi$, and then multiply by 2 , where $\phi$ is half the angle subtended by the whole filament.

Since

$$
\int_{0}^{\phi} \cos ^{2} \beta d \beta=\frac{\sin \phi \cos \phi}{2}+\frac{\phi}{2}
$$

and since

$$
l \cos \phi=\mathrm{D} \sin \phi \text {, }
$$

we see that

$$
2 \int_{0}^{\phi} \frac{\mathrm{I}_{\mathrm{H}} \mathrm{D} \cos ^{2} \beta d \beta}{\mathrm{D}^{2}}=\frac{\mathrm{I}_{\mathrm{H}}}{\mathrm{J}^{\ddot{2}}} 2 l\left(\frac{\cos ^{2} \phi}{2}+\frac{\phi \cot \phi}{2}\right) .
$$

Hence the correcting factor when photometering a filament, the apparent length $2 l$ of which subtends a finite angle $2 \phi$ at the photometer-disk, is $\frac{1}{2}\left(\cos ^{2} \phi+\phi \cot \phi\right)$. Accordingly the ratio of mean spherical to mean horizontal candle-power in this last case is given by the expression

$$
\frac{\mathrm{I}_{\mathrm{S}}}{\mathrm{I}_{\mathrm{II}}}=\frac{\pi}{4}\left\{\frac{2}{\cos ^{2} \phi+\phi \cot \phi}\right\} .
$$

If the filament is not a straight filament but a loop as in $\mathrm{P} 2$ 


\section{Dr. Fleming: Ratio betueen Mean Spherical and}

an actual lamp, which may be so coiled that the different portions lie in different planes, we have an additional small correction to make. We may first notice the effect of the inclination of a straight filament in various directions.

Let three coordinate planes be drawn through the centre of the sphere of reference. Let one of these planes be horizontal. Let the photometer-disk be placed with its plane vertical. Let two other planes be described through the centre of the sphere which are respectively parallel and perpendicular to the photometer-disk plane.

Then consider a straight filament lying in the plane, perpendicular to the photometer-disk and having an inclination to it.

It is clear that if the cosine law of radiation holds good, the light sent out by each element of the inclined filament in a horizontal direction will be the same as that of its projection on a plane parallel to the photometric disk. Hence we may substitute for the inclined filament its projection on a vertical plane, and if we assume the filament and its projection have the same candle-power per unit of lengtb, the horizontal illuminating power will be the same in the two cases. If, therefore, the filament lies in any plane we may consider it divided into equal elements of length, each of which makes its own contribution to the mean spherical and the mean horizontal candle-power. Disregarding for the moment the effect of distance from the horizontal plane on the illtiminating effect of each element on the photometer-disk, we see that the ratio of the mean spherical to the mean horizontal candle-power must be greater for each element of length of the filament the more it is inclined to the vertical plane or the less its inclination to the line joining it to the photometerdisk.

Hence if the whole filament lies in one plane placed perpendicularly to the photometer axis the ratio $\mathbf{I}_{\mathbf{S}} / \mathbf{I}_{\mathbf{H}}$ must be $\pi / 4$. If it does not lie wholly in that plane the ratio will be somewhat greater.

In the case of filaments coiled in various ways a small correcting factor is also necessary, providing for the reduced illuminating effect of each equal element of the filament, into which the filament may be considered to be divided, the further the element is removed from the axis of the photometer, that is, from the horizontal plane. In the case of simple horseshoe-shaped filaments, so piaced that the whole filament is visible, this correcting factor will be the factor

$$
\frac{2}{\cos ^{2} \phi+\phi \cot \phi}
$$


where $\phi$ is half the angle subtended by the whole filament at the centre of the photometer-disk.

If the filament has a double or multiple loop, then the precise correcting factor for angular magnitude of the whole loop is not quite so sharply defined. It will, however, always operate to increase the fraction or percentage which represents the ratio of mean spherical to mean horizontal candle-power. It will always be a small correction if the whole filament subtends an angle, say, of not more than $10^{\circ}$ at the centre of the photometer-disk, as in the ordinary photometry of an incandescent electric lamp.

These considerations show that incandescent lamp manufacturers can without much difficulty furnish the figures required to give the true photometric efficiency of any type of lamp. Instead of stating, as at present, the value of the maximum candle-power taken in one direction, viz., the direction perpendicular to the axis of the lamp in which the whole of the filament is seen exposed, they should furnish the mean horizontal candle-power taken in the same direction when rotating the lamp around its vertical axis, using for this purpose well-known mechanical devices.

If this reading is taken at such a distance from the lamp that the whole filament does not subtend a greater angle than $5^{\circ}$ to $10^{\circ}$ at the photometer-disk, then the mean spherical candle-power of the incandeseent lamp is for practical purposes very nearly obtained by multiplying this mean horizontal candle-power by the factor $\pi / 4$. From the measurement of the electric power in watts supplied to the lamp, we have at once the means of calculating the total flux of light per watt which fixes scientifically the efficiency of the lamp as an energy-transforming device.

\section{APPENDIX.}

To enable a judgment to be made as to the validity of the assumption that the intensity of the light proceeding from a filament in any direction varies as the cosine of the angle between the ray and the normal to the element, the following experiments were tried by Mr. G. B. Dyke at the suggestion of the writer.

A tubular incandescent carbon-filament lamp was prepared with a straight filament about 10 inches in length. This was placed on a photometric gallery at a distance of about 40 inches from a Lummer-Brodhun photometer, and two baffle-screens interposed having circular holes in them about 1 inch in diameter. These screens were placed so as to divide the 


\section{Dr. Fleming: Ratio between Mean Spherical and}

space between the photometer and the lamp into three nearly equal parts.

The tubular lamp had a protractor attached to it, and could be tilted over at various angles to the vertical line, keeping it in one vertical plane normal to the photometer-disk. It is obvious that if the illumination sent out from unit of length of the straight filament obeys the cosine law above mentioned, then tilting the lamp as described to various angles should make no difference in the observed illumination on the photometer. As a matter of fact, the observed illumination decreased rapidly as the lamp was tilted over, in accordance with the figures in the following table.

$\begin{array}{cc}\begin{array}{c}\text { Angle between the straight } \\ \text { tilament and the plumb-line }\end{array} & \begin{array}{c}\text { Observed candle-power or } \\ \text { illumination through the } \\ \text { two baffle-screens. }\end{array} \\ \text { through its centre. } & 2 \cdot 33 \\ 0 & 2 \cdot 27 \\ 15 & 2 \cdot 23 \\ 30 & 2 \cdot 15 \\ 45 & 1 \cdot 96 \\ 60 & 1 \cdot 06\end{array}$

It is clear, however, that some part of the decrease of light at high inclinations is due to the loss of light by reflexion at the inner surface of the tubular glass bulb. To obtain the correction for this reflexion a sheet of glass was interposed between an ordinary lamp and the photometer, the plane of the glass being tilted at various angles to the plane normal to the ray. The photometric readings were as follows :-

Angle at which sheet of glass was placed to the plane normal to the ray.

$\begin{array}{cc}\text { mal to the ray. } & \text { of the light. } \\ 0 & 26 \cdot 1 \text { c.p. } \\ 15 & 25 \cdot 9 " \\ 30 & 25 \cdot 8 " \\ 45 & 25 \cdot 2 " \\ 60 & 25 \cdot 4 " \\ 75 & 16.7 \%\end{array}$

It follows therefore, that if the carbon filament in the tubular lamp could have been used without a glass envelope, the light sent by it through the baffle-screens would have been greater in the ratio of the first term in the right-hand column of the previous table to any succeeding term in the same column corresponding to the same angle of inclination of the filament.

If therefore we call the intensity of the light sent through the screens unity when the filament is vertical, then its true 
intensity corrected for reflexion when the lamp is tilted to various angles is as follows:-

Angle of

Inclination.

8

15

30

45

60

75
Corrected Value of Intensity of

Licht emitted per unit of length.

$2 \cdot 33 \times 26 \cdot 1 \div 2 \cdot 33 \times 26 \cdot 1=1 \cdot 00$

$2 \cdot 27 \times 26 \cdot 1 \div 2 \cdot 33 \times 25 \cdot 9=\cdot 98$

$2 \cdot 33 \times 26 \cdot 1 \div 2 \cdot 33 \times 25 \cdot 8=1 \cdot 00$

$2 \cdot 15 \times 26 \cdot 1 \div 2 \cdot 33 \times 25 \cdot 2=\cdot 96$

$1.96 \times 26 \cdot 1 \div 2 \cdot 33 \times 25 \cdot 4=\cdot 86$

$1 \cdot 06 \times 26 \cdot 1 \div 2 \cdot 33 \times 16 \cdot 7=\cdot 71$

The figures in the last column therefore represent the intensity of the light sent out by unit length of the incandescent filament at various angles of the normal. If the cosine law were strictly fulfilled these values should all be unity.

The easiest way to determine the effect of this departure from the true cosine law on the mean spherical candle-power, is to describe a semicircle (firm line) of unit radius and to set off on its diameter distances from the centre $\mathrm{C}$ (see fig. 3)

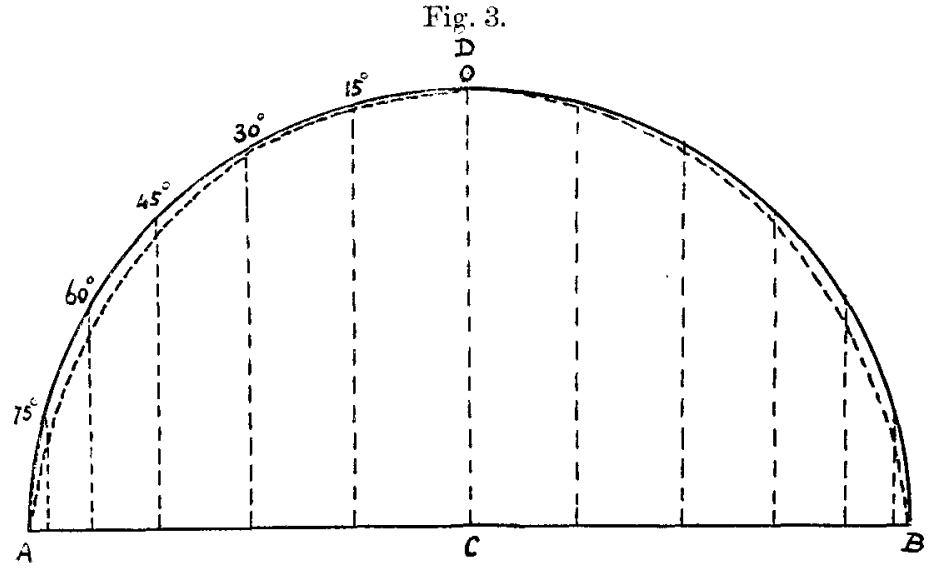

proportional to the sines of the angles $0^{\circ}, 15^{\circ}, 30^{\circ}$, \&c., and then through these points to draw ordinates (dotted lines) to the diameter of the semicircle. Fractions of these ordinates equal to the fractions in the last column of the above table are then set off, and the upper ends of these define a curve (dotted line) which is the Rousseau diagram of the photometric curve of luminous radiation of the filament.

If the value of the mean ordinate of this last (dotted) curve is taken, it gives us the ratio of the mean spherical to the mean horizontal candle-power of the straight filament. For the (dotted) curve as drawn in fig. 3 delineating the observations made with the above-mentioned carbon filament, this 
mean ordinate or corrected ratio comes out 0.77 instead of 0.785 as it should do if the cosine law had been strictly fulfilled. In this case the dotted line would have fallen on the semicircle and coincided with it.

Another similar set of measurements was made with a straight oxide filament taken from a Nernst lamp used without heating-coil, and with the supports of the filament all carefully painted dead-black to avoid errors due to reflected light. In this last case no correction due to loss of light by reflexion from a glass envelope was needed, as the Nernst filament was used in the open air. The ratio of the mean spherical to mean horizontal filament in this last case was experimentally found to be $0 \cdot 785$, or exactly the ralue it should have if the cosine law is obeyed.

We have therefore in these experiments a justification for the assumption made in this paper for the law of luminous radiation from a straight incandescent filament.

My thanks are due to Mr. Dyke for his assistance in the experimental portion of this work.

XXVII. On the Flux of Light from the Electric Are with Varying Power-Supply. By G. B. Dчкe, B.Sc.*

[Plate II.

TN 1896 a paper was communicated to the Phycical Society 1 by Dr. J. A. Fleming, F.R.S., and Mr. J. E. Petavel †, recording the results of numerous observations on the Electric Arc, dealing, amongst other matters, with the question of the relation between the flux of light, or mean spherical candlepower, and the watts expended in the arc.

The time and labour necessary to obtain the mean spherical candle-power of any source of light from observations made on the point-by-point method then employed, prevented any very extended study of this relation.

The construction of the integrating photometer, described by the author in his paper "On the Practical Determination of the Mean Spherical Candle Power of Incandescent and Arc Lamps " $\ddagger$, having, to a very large extent, removed these difficulties, it was tbought that a more extended series of observations in this direction might be undertaken, and facilities for this purpose were kindly granted in the Pender

* Communicated by the Physical Society : read March 24, 1905.

+ "An Analytical Study of the Alteriating-Current Are," by $J$. A. Fleming, M.A., 'D.Sc., F.R.S., and G. E. Petavel, Phil. Mag. April 1896, p. 315 .

" On the Practical Determination of the Mean Spherical CandlePower of Incaudescent and Arc Lamps," by G. B. Dyke, B.Sc., Phil. MIg. Jan. 1905, p. 136, and Proc. Phys. Soc. vol. xix. 